\title{
Vitamin-D Deficiency among Non-Diabetic and Type-2 Diabetic Individuals: A Comparative Study
}

ARTI SAURABH MULEY', UMA MAHADEVAN IYER²

\section{ABSTRACT}

Introduction: Vitamin-D Deficiency (VDD) is widespread across the globe irrespective of age, gender, race, location or disease profile. Even in a sun drenched country like India it is highly prevalent and now recognised as a public health problem.

Aim: To map the prevalence of VDD among non-diabetic and Type-2 Diabetes Mellitus (T2DM) adults residing in a city of western India, and to examine the risk factors associated with it.

Materials and Methods: Type- 2 diabetic $(n=114)$ and nondiabetic $(n=129)$ adults of age $30-65$ years were enrolled for this exploratory study from a diabetic clinic and from different localities of the city respectively. Anthropometric measurements like height, weight, Waist-Circumference (WC) and Waist-Stature Ratio (WSR) were recorded using standard methods. Fasting blood sample was drawn to estimate biochemical parameters in a certified laboratory. Serum $25(\mathrm{OH}) \mathrm{D}$ was estimated by Chemiluminescence Immunoassay (CLIA) technique. Lipid profile \{Total Cholesterol (TC), Triglycerides (TAG), LowDensity Lipoprotein (LDL) and High-Density Lipoprotein (HDL)\} was estimated using enzymatic kits by Photometry. The high sensitivity assay of serum C-Reactive Protein (hs-CRP) was done using nephelometry. T-test, chi-square, Pearson ' $r$ ' value and linear regression was computed using SPSS version-20 for statistical analysis.

Results: The mean serum 25(OH)D level among non-diabetic and T2DM participants $(13.7 \pm 7.2$ and $14.2 \pm 8.8 \mathrm{ng} / \mathrm{mL}$, respectively) was much lower to the recommended level of $30 \mathrm{ng} / \mathrm{mL}$. In both the populations about $88 \%$ of the participants were found to be VDD with serum 25(OH)D levels $<20 \mathrm{ng} / \mathrm{mL}$. WC, WSR, BMI, percent body fat and lipid parameters- TC and LDL-cholesterol showed a negative significant correlation with the vitamin-D levels among the diabetic patients. While among the non-diabetics only percent body fat and LDL-cholesterol correlated significantly. Indices of central obesity and LDLcholesterol emerged as common and significant suppressors of optimum vitamin-D status among both the groups.

Conclusion: A high prevalence of VDD was observed among the participants. Hence vitamin-D screening should be done in routine medical check-ups, supplementations, and vitamin D-fortified foods should be encouraged for these groups. Healthy lifestyle changes should be propagated and adopted to prevent as well as manage the metabolic alterations among the population due to VDD.

\section{INTRODUCTION}

Vitamin-D, also known as the sunshine vitamin, has received a lot of attention in recent times as a result of a meteoric rise in the prevalence of its deficiency. VDD is now recognised as a global pandemic with the major cause being the lack of appreciation that sun exposure has been and continues to be the major source of vitamin-D for people of all ages. Serum 25-hydroxyvitamin D $(25(\mathrm{OH})$ D) value is a generally accepted indicator of vitamin-D status. It has been estimated that about 1 billion people worldwide are vitamin-D deficient or insufficient based on their serum 25(OH)D levels [1]. Studies conducted in India among various socio-economic groups, different ages, and both genders as well as different disease states, report widespread VDD/insufficiency in the country $[2,3]$. The prevalence ranges from $70-90 \%$ among healthy adults and pre and post-menopausal women in India [4-6]. The individual's behaviour to avoid sunlight or clothing which prevents exposure to sunlight and low availability as well as intake of vitamin-D rich foods are thought to be the probable reasons. Vitamin-D has shown to play a crucial role in a large amount of physiological functions. VDD is also associated with many acute and chronic illnesses including disorders of calcium metabolism, autoimmune diseases, some cancers like of bladder, colon, breast, etc., type-2 diabetes mellitus, cardiovascular disease and infectious diseases [7]. Though the exact pathways are not clear yet, with the rise in incidences of these conditions it is important to identify the determinants of VDD among the high risk population. Recent evidence shows that vitamin-D status is important to regulate some pathways related to diabetes development, thus making VDD more evident in diabetic patients. A review indicated that VDD may predispose to glucose intolerance, altered insulin secretion and T2DM [8], either through a direct action via Vitamin-D Receptor (VDR) activation or indirectly via calcaemic hormones and also through inflammation [9]. There is compelling evidence that low vitamin-D levels are also associated with classic cardiovascular risk factors like obesity and hypertension, which are prominent co-morbidities among T2DM patients [10]. Also, there still remains some controversy regarding what level of serum $25(\mathrm{OH})$ $\mathrm{D}$ should be attained for both bone health and reducing risk for VDD associated with acute and chronic diseases [11]. Currently, it is a common practice by physicians in India to prescribe a cholecalciferol sachet of $1500 \mu \mathrm{g}(60,000$ International Units [IU]) to be taken each week for 6-8 weeks for overt or occult VDD among the general population [12]. Other treatment options may include vitamin-D fortification in staple foods or supplementation of vitamin-D along with calcium in form of syrups or tablets.

A study among randomly selected 299 normal individuals (age 18-75 years) in Secunderabad, Telangana, India, found $81.6 \%$ to be vitamin-D deficient. $34.4 \%$ had $25(\mathrm{OH}) \mathrm{D}$ deficiency as well as metabolic syndrome [13]. Muley A et al., in their previous study reported that $88 \%$ of the apparently healthy participants between age of 30-60 years had VDD, with significantly higher rates among 
females than males (95.1 vs $76.6 \%, p<0.01$ ). Being a female $(\mathrm{OR}=5.95 ; 95 \% \mathrm{Cl}$ 1.77-19.99, $\mathrm{p}<0.01)$ and having high waist circumference $(\mathrm{OR}=3.55 ; 95 \% \mathrm{Cl} 1.1-11.2, \quad \mathrm{p}<0.05)$ showed significantly higher odds for low vitamin-D (<20 ng/mL) [14]. A recently conducted Pan India study among adults with mean age $52.9 \pm 12.49$ years, reported an overall prevalence of VDD and insufficiency as $83.7 \%$. Prevalence of low vitamin-D levels amongst patients with T2DM $(n=500), H T(n=499)$ and T2DM+HT $(n=502)$ was also very high (84.2\%, 82.6\% and $84.5 \%$ respectively) [15].

This shows that studies do exist among various groups, but there is still paucity of research comparing vitamin-D levels among apparently healthy and type-2 diabetic patients in India, especially in the western regions. Thus, the present study was planned with the primary objective to map the prevalence of VDD among the T2DM and non-diabetic patients residing in western India and to identify the various risk factors associated with their vitamin-D status.

\section{MATERIALS AND METHODS}

The present research was a cross-sectional comparative study between apparently healthy individuals $(n=129)$ and type-2 diabetic patients $(n=114)$ residing in Vadodara, Gujarat, India.

\section{Selection of Participants}

For the enrolment of participants residing in different localities of the city, the city was divided into five zones- north, south, central, west \& east. Through snow ball method 129 participants; 26 each from north, south, east and west zone, while 25 were enrolled from the central zone. A diabetic clinic located in the central zone was purposively selected to enrol T2DM patients from all the zones of the city visiting it. The detail procedure of participant enrolment is explained in [Table/Fig-1]. No drop outs were reported during the course of study. The study was carried out for a period of seven months from September 2012 to April 2013.

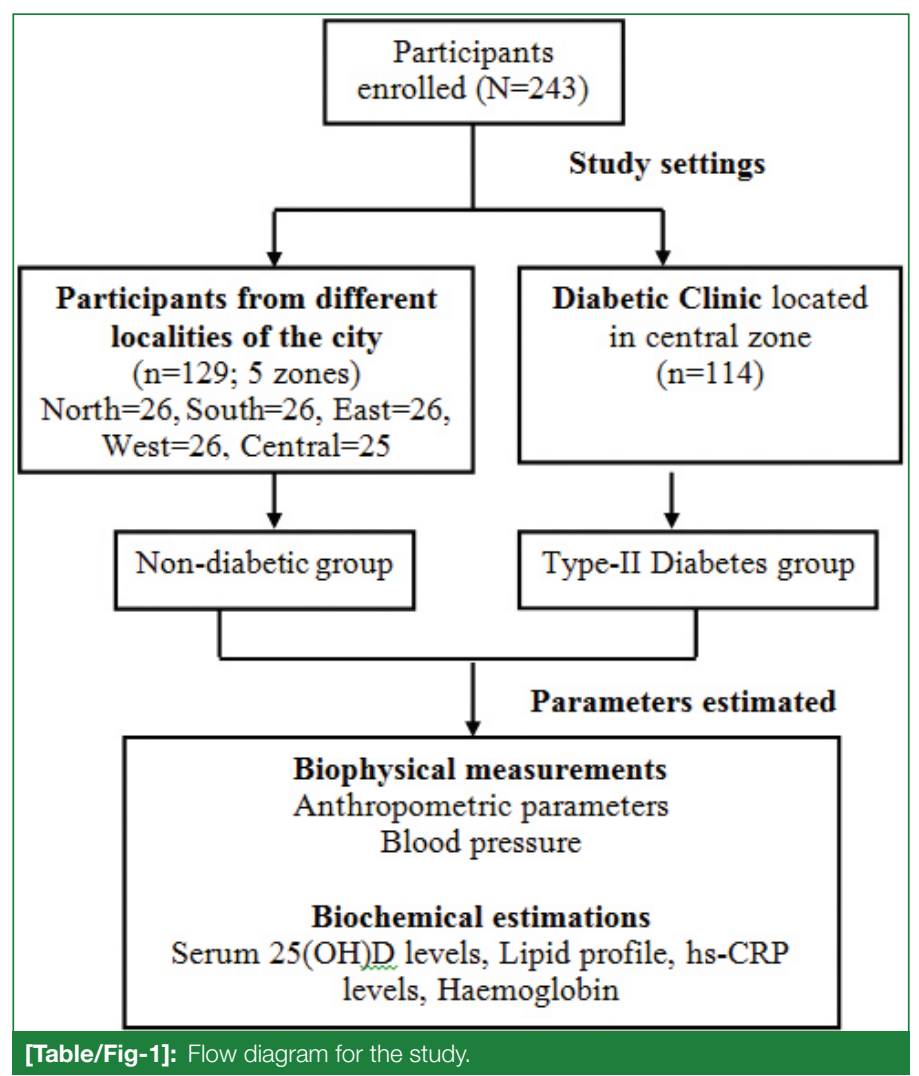

\section{Sample Size Calculation}

From the available literature, the prevalence of VDD ranges from 70 to $90 \%$ in India [4-6]. Hence for this study a sample size of 138 was calculated, taking the prevalence of VDD as $80 \%$, at 80 power of the study and $95 \%$ confidence interval. Thus the sample size of this study ( $n=243$ ) was adequate to identify the determinants of vitamin-D status of the population.

\section{Inclusion-Exclusion Criteria}

The inclusion criterion was-participants should be in the age group 30-65 years; they should be residents of the city and give a positive consent to participate in the study and for the blood estimations as well. Pregnant women and lactating mothers, those taking vitamin-D supplements, those suffering from gestational or type-1 diabetes were excluded.

\section{Ethical Approval}

The study was approved by the Institutional Ethics Committee for Human Research (Reference No. IECHR/2013/04) which is responsible for ethical issues in all research projects involving human subjects conducted by the department. Written informed consent was taken from all the participants in English as well as local language (Gujarati).

\section{Data Collection}

Information regarding socio-demographic characteristics, medical history and medications was collected using a pre-tested semistructured questionnaire. Anthropometric measurements were also recorded. Weight was taken using calibrated Salter electronic bathroom scale. Height, waist and hip circumference were measured using non-stretchable fibre glass tape to the nearest $0.1 \mathrm{~cm}$. The waist was defined as the point midway between the iliac crest and coastal margin and hip circumference as the widest circumference over the buttocks and below the iliac crest $[16,17]$. Percentage body fat was calculated using the Omron body fat monitor (Model HBF-306). All measurements were taken by trained researchers. $\mathrm{BMI}$ was calculated by standard formula of weight in $\mathrm{kg} / \mathrm{height}^{2}$ in meters. Blood pressure was measured by clinically validated digital BP meter (Omron HEM-7203 model). Three measurements were taken and an average of the readings was considered.

After an over-night fast, venous blood samples were collected by a certified technician to carry out the biochemical estimations. Serum $25(\mathrm{OH}) \mathrm{D}$ was estimated by Chemiluminescence Immunoassay (CLIA) technique to ascertain the vitamin-D status of participants. This was used as the serum concentration of $25(\mathrm{OH}) \mathrm{D}$ is widely regarded as a "gold standard" indicator of vitamin-D status as it is a good reflection of cumulative exposure to sunlight and dietary intake of vitamin-D [18]. VDD was defined as serum $25(\mathrm{OH}) \mathrm{D}$ concentration of $<20 \mathrm{ng} / \mathrm{mL}$ and categorized as insufficiency $(20-\leq 30 \mathrm{ng} / \mathrm{mL})$ and sufficiency (>30 ng/mL) [19]. Lipid profile which included Total Cholesterol (TC), Triglycerides (TAG), Low-Density Lipoprotein (LDL-C) and High-Density Lipoprotein (HDL-C) was estimated using enzymatic kits. Instrumentation used for the analysis was Olympus AU2700 and Advia 1800 and the detection technology was by Photometry. Various ratios like TC/HDL, TAG/HDL and LDL/ $\mathrm{HDL}$ were calculated using Microsoft excel. Atherogenic index of plasma were calculated by taking log 10 (TAG/HDL). Haemoglobin was estimated by cyanmethemoglobin method in autoanalyser, HsCRP by Nephelometry. These biochemical analyses were estimated at an ISO 9001:2008, NABL (India) and CAP (College of American Pathologist) certified laboratory. Metabolic Syndrome (MS) was defined based on two criteria- the International Diabetes federation (IDF) classification, 2005 and the guidelines given by Adult Treatment Panel-III in 2001 [20].

\section{STATISTICAL ANALYSIS}

Data was entered in Microsoft Excel 2007 and verified by the researcher. Statistical Package for Social Sciences (SPSS) version 20 was used for statistical analysis of the data. A two-tailed $\mathrm{p}$-value was used for calculating statistical significance; a value of $p<0.05$ was taken to be significant. For quantifiable variables, 
descriptive statistics (means and standard deviation) were calculated. Comparison of means was done using independent t-test for non-diabetic and diabetic populations. Chi-square value was computed for checking the significance for categorical variables. Pearson ' $r$ ' value was calculated to study the correlations between vitamin-D levels and risk factors for both the populations. To identify the predicators of serum vitamin-D status a stepwise multivariate analysis (Linear regression) for both the populations was carried out.

\section{RESULTS}

The mean serum 25(OH)D levels in both the diabetic and nondiabetic populations was less than $20 \mathrm{ng} / \mathrm{mL}(14.2 \pm 8.8$ and $13.7 \pm 7.2$, respectively), indicating the presence of VDD among the participants. When the subjects were divided into various categories of VDD based on their serum 25(OH)D levels, it was observed that in both the populations about $88 \%$ of the subjects were vitamin-D deficient as depicted in [Table/Fig-2]. Only about 4\% of the nondiabetics and $5 \%$ of the diabetics were found to be vitamin-D sufficient with serum levels more than $30 \mathrm{ng} / \mathrm{mL}$. Thus, irrespective of the presence or absence of diabetes, a high prevalence of VDD was observed among the participants.

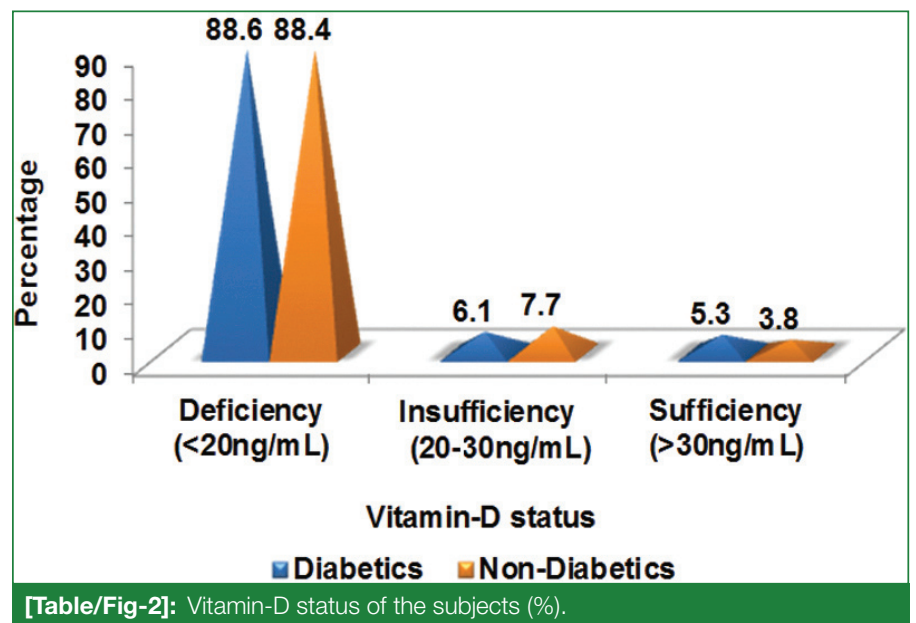

In context to lipid profile, the prevalence of hypertriglyceridemia, inflammation, altered atherogenic indices and atherogenic index of plasma was significantly higher in the diabetic population as compared to non-diabetic participants which is shown in [Table/Fig-3]. The diabetic patients thus posed a higher risk for CVDs which is a known and accepted fact. Metabolic syndrome (MS) which was defined by two criteria and according to both; the diabetic population had significantly higher prevalence as compared to the non-diabetic population [Table/Fig-4].

The anthropometric measurements- WC $(p<0.05)$, WSR $(p<0.01)$, BMI $(p<0.05)$, percent body fat $(p<0.05)$ and lipid parameterstotal cholesterol $(p<0.05)$ and LDL-C $(p<0.01)$ showed a negative significant correlation with the vitamin-D levels among the diabetic subjects, whereas among the non-diabetic subjects only percent body fat $(p<0.01)$ and LDL-C $(p<0.05)$ were negatively significantly correlated. Thus, from this analysis it was observed that only percent body fat and LDL-C were the variables showing significant correlation with the vitamin-D levels of the subjects in both the populations. As seen in [Table/Fig-5], both the parameters showed a negative correlation, hence suggesting that abnormal elevated levels of these would compromise vitamin-D levels among the subjects.

The stepwise multivariate analysis revealed that for the diabetic group; WSR and LDL-C were the significant predictors. While among the non-diabetics; percent body fat and LDL-C emerged as the predictors (refer [Table/Fig-6]). Though only LDL-C was the common predictor between the populations, it is to be noticed that the other predictors were related to abdominal obesity. Hence, it is very important to monitor them closely and adopt measures to prevent obesity.

\begin{tabular}{|l|c|c|c|}
\hline Parameter & $\begin{array}{c}\text { Diabetics } \\
(\mathrm{n}=114)\end{array}$ & $\begin{array}{c}\text { Non-diabetics } \\
(\mathrm{n}=129)\end{array}$ & $\chi^{2} \mathrm{p}$-value \\
\hline $\mathrm{TC} \geq 200 \mathrm{mg} / \mathrm{dL}$ & $40(35.1)$ & $53(41.1)$ & 0.337 \\
\hline $\mathrm{TAG} \geq 150 \mathrm{mg} / \mathrm{dL}$ & $40(35.4)$ & $25(19.4)$ & $0.005^{\star *}$ \\
\hline $\mathrm{LDL}-\mathrm{C} \geq 100 \mathrm{mg} / \mathrm{dL}$ & $72(64.9)$ & $83(64.3)$ & 0.933 \\
\hline $\begin{array}{l}\mathrm{HDL}-\mathrm{C}<40 \mathrm{mg} / \mathrm{dL}(\mathrm{Male}) \\
<50 \mathrm{mg} / \mathrm{dL}(\text { Female) }\end{array}$ & $57(50.0)$ & $54(41.9)$ & 0.204 \\
\hline $\mathrm{TC} / \mathrm{HDL} \geq 5$ & $22(19.3)$ & $12(9.3)$ & $0.025^{\star}$ \\
\hline TAG/HDL $\geq 3$ & $57(50.4)$ & $30(23.3)$ & $<0.001^{\text {*** }}$ \\
\hline LDL/HDL $\geq 3.5$ & $11(9.9)$ & $3(2.3)$ & $0.012^{\star}$ \\
\hline
\end{tabular}

Atherogenic Index of Plasma (AIP)

\begin{tabular}{|c|c|c|c|}
\hline$<0.11$ (low risk) & $3(2.7)$ & $24(18.6)$ & \multirow{3}{*}{$<0.001^{\star \star \star}$} \\
\hline $0.11-0.21$ (moderate risk) & $10(9.0)$ & $16(12.4)$ & \\
\hline$>0.21$ (high risk) & 98 (88.3) & $89(69.0)$ & \\
\hline
\end{tabular}

Inflammatory marker hsCRP

\begin{tabular}{|c|c|c|c|}
\hline$>01 \mathrm{ma} / \mathrm{dl}$ & $90(789)$ & $80(600)$ & $<0,001^{* * *}$ \\
\hline
\end{tabular}

[Table/Fig-3]: Prevalence of hyperlipidemia and inflammation among the subjects. (n, \%)

TC: Total cholesterol; TAG- Triglyceride; LDL-C: Low density lipoprotein-cholesterol; HDL-C: High density lipoprotein-cholesterol; HsCRP: High sensitivity C-reactive protein

$p<0.001^{\star \star *},<0.01^{* *},<0.05^{\star}$ Values in parenthesis indicate percent

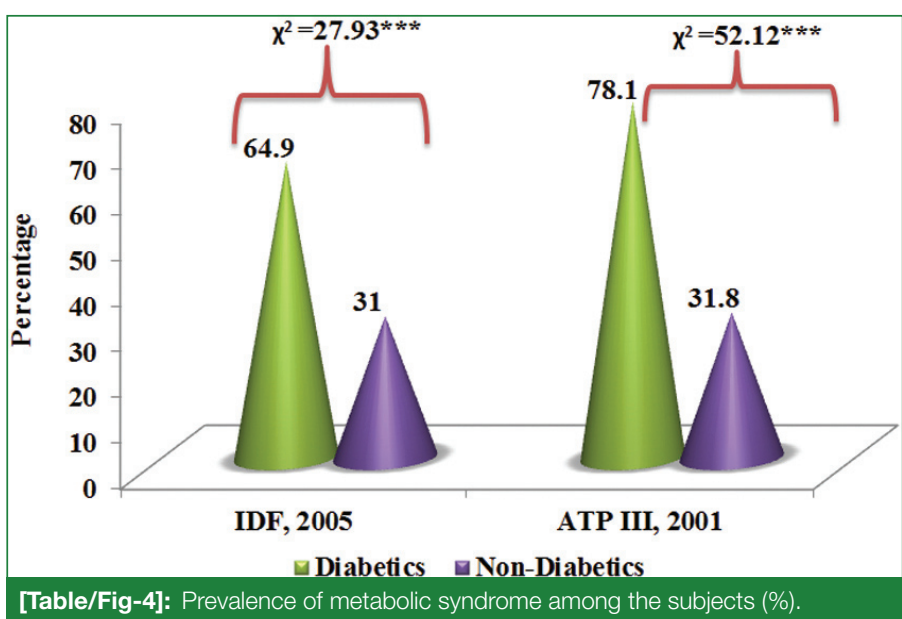

[Table/Fig-4]: Prevalence of metabolic syndrome among the subjects (\%).

\begin{tabular}{|c|c|c|}
\hline \multirow[b]{2}{*}{ Variables } & Diabetics $(n=114)$ & Non-diabetics $(n=129)$ \\
\hline & \multicolumn{2}{|c|}{ Pearson ' $r$ ' value } \\
\hline WC (cm) & $-0.235^{\star}$ & \\
\hline WSR & $-0.266^{\star \star}$ & \\
\hline $\mathrm{BMl}$ & $-0.212^{*}$ & \\
\hline Body fat (\%) & $-0.237^{*}$ & $-0.246^{\star *}$ \\
\hline Total cholesterol & $-0.206^{\star}$ & \\
\hline LDL cholesterol & $-0.247^{\star \star}$ & $-0.184^{*}$ \\
\hline
\end{tabular}

\begin{tabular}{|l|c|c|c|}
\hline \multicolumn{2}{|c|}{ Diabetics $(\mathrm{n}=114)$} & \multicolumn{2}{c|}{ Non-diabetics $(\mathrm{n}=129)$} \\
\hline Predictor variables & $\beta$-coefficient & Predictor variables & $\beta$-coefficient \\
\hline WSR & $-0.255^{\star *}$ & $\%$ Body fat & $-0.200^{\star}$ \\
\hline LDL-C & $-0.210^{\star}$ & LDL-C & $-0.168^{\star}$ \\
\hline
\end{tabular}
$\begin{aligned} & \text { [Table/Fig-6]: Summary of predictors variables of vitamin-D status. } \\
& \text { (Stepwise Forward Linear Regression). } \\
& p<0.05^{*},<0.01^{\star *}\end{aligned}$

\section{DISCUSSION}

VDD is considered pandemic now. Alarming prevalence has been reported in various groups of individuals. Past literature also suggests a deleterious effect of low vitamin-D on general health, proposing an association between hypovitaminosis-D and the aetiology and progression of T2DM. However, there is felt need to have a comparative analysis of vitamin-D status and its probable determinants among healthy individuals and patients with medical 
conditions. Hence, the present study was conducted to compare non-diabetic and T2DM patients with respect to their vitamin-D status and related risk factors.

Due to the role of vitamin-D in many of the pathways related to glucose metabolism, it is not wrong to assume that patients with T2DM are more prone to its deficiency. However, in the present study a high prevalence of VDD (about 88\%) was observed in both the diabetic as well as non-diabetic subjects. In a cross-sectional study, conducted in Iran, similar high prevalence of $83.3 \%$ and $75.6 \%$ was reported among 100 type-2 diabetic patients and 100 healthy subjects aged between $20-80$ years respectively. It was further reported that the serum $25(\mathrm{OH}) \mathrm{D}$ levels $(22.08 \pm 15.20$ vs 22.22 $\pm 10.03, p=0.75)$ and $B M l(26.22 \pm 9.30$ vs $26.26 \pm 4.55$, $\mathrm{p}=0.98$ ) were comparable between diabetic subjects and healthy controls and that serum concentration of $25(\mathrm{OH}) \mathrm{D}$ correlated inversely with BMI in diabetic patients and healthy controls [21]. In the present study however, BMI showed significant negative correlation only among the diabetic subjects $(28.1 \pm 5.3$ vs $25.9 \pm 4.7$, $\mathrm{p}<0.001)$. Similarly, in a case-control study among 58 type-2 diabetic patients and 58 non-diabetic controls of Gaza strip, the reported prevalence was significantly higher among the diabetics as compared to controls (70.7\% vs $\left.32.8 \% ; \chi^{2}=14.672, p<0.001\right)$ [22]. Subramanian A et al., also in his study among 92 T2DM patients and equal number of non-diabetic patients in northern India reported VDD to be significantly more prevalent among T2DM patients than the non-diabetic patients (57.6\% vs 33.3\%, p=0.001) [23]. Though these studies differ to our findings in terms of percent prevalence, they do point towards presence of VDD among both the diabetics and non-diabetics.

Vitamin-D plays an indirect but an important role in carbohydrate and lipid metabolism as reflected by its association with type-2 diabetes, MS and obesity. In apparently healthy population also hypovitaminosis-D results in non-musculoskeletal consequences including increased risks of chronic diseases such as T2DM, Cardiovascular Disease (CVD), hypertension and obesity [24]. Hence, an attempt was made to compare the presence of obesity and dyslipidemia among the groups and determine the determinants of vitamin-D status. In the present study, the diabetic population showed a higher prevalence of MS as compared to their non-diabetic counter parts. This trend is obvious, as diabetics are more prone to unfavourable alterations in their cardio-metabolic parameters due to physiological changes because of the presence of the disease. However, it is a matter of concern as this indicates the presence of multiple risk factors among the participants.

Also, the prevalence of obesity in the present study was found to be significantly higher among the diabetic population. WSR and percent body fat; both indicators of abdominal obesity emerged as significant predictors for poor vitamin-D status among the subjects in both groups. A recently conducted meta-analysis also analysed an overall inverse relationship between serum vitamin-D status and $\mathrm{BMl}$ in studies of both diabetic $(r=-0.173,95 \%=-0.241$ to -0.103 , $p<0.001)$ and non-diabetic $(r=-0.152,95 \%=-0.187$ to -0.116 , $\mathrm{p}<0.001$ ) subjects [25].

Diabetics are more prone to CVDs and show unfavourable alterations in the lipid levels due to metabolic changes. In the present study, prevalence of hyperlipidemia was high among the diabetic subjects with significant levels for hypertriglyceridemia, altered atherogenic indices and inflammation. Maged MY et al., in his study has reported that serum glycated haemoglobin $(\mathrm{HbA} 1 \mathrm{c})$ and triglycerides were significantly higher in diabetic patients than in controls whereas serum HDL-cholesterol was significantly lower in patients [22]. However, in another cross-sectional study, among 235 apparently healthy women and men aged 20-60 from the city of Ahvaz in Iran, $94 \%$ of the participants had VDD $(25(\mathrm{OH}) \mathrm{D}<50 \mathrm{nmol} / \mathrm{L})$ and the subjects with hypovitaminosis-D were almost 4.38 times more likely to be overweight/obese compared with subjects with vitamin-D sufficiency (OR:4.38, $p=0.011)$. But, there was no significant association found between serum $25(\mathrm{OH}) \mathrm{D}$ concentration and the lipid profile components [26]. Thus though the data points out towards role of vitamin-D sufficiency in maintaining favourable glycaemic and lipemic profile among type-2 diabetics, well planned randomized trials are required to arrive at concrete conclusions.

\section{LIMITATION}

The study had few limitations. Firstly only one diabetic clinic was selected. Though it was centrally located but in some sense the zonal enrolment of the participants was restricted. Secondly the data was collected few years back. Though not outdated, it could have been more useful if published earlier.

\section{CONCLUSION}

The high prevalence of VDD observed in both the populations clearly indicates that vitamin-D estimation should be included in routine body check-ups irrespective of the presence or absence of any disease condition. It is also recommended that necessary efforts should be put in to manage central obesity and ideal lipid levels by the population, as these were identified as significant predictors for poor vitamin-D levels. This can be done by adopting healthy lifestyle changes such as regular exercise and daily exposure to sunlight for atleast 30 minutes, which in turn will help to increase the cutaneous production of vitamin-D in the body. Lastly policies for vitamin-D supplementation and food fortification strategies should be framed and implemented in India to make vitamin-D rich food options available to the population, which will help to curb the high deficiency rate.

\section{ACKNOWLEDGEMENTS}

The authors would like to acknowledge all the participants for giving consent to be a part of this study. The authors also thank Dr. Mona Shah, Consulting Diabeteologist and Endocrinologist, Vadodara for enrolment of diabetic patients from her clinic.

Source of funding: The study was funded by University Grants Commission (UGC), New Delhi in the form of research fellowship to corresponding author.

\section{REFERENCES}

[1] Holick MF. Vitamin D deficiency. N Engl J Med. 2007;357:266-81.

[2] Harinarayan CV, Joshi SR. Vitamin D status in India- its implications and remedial measures. J Assoc Physicians India. 2009:57:40-48.

[3] Harinarayan CV, Gupta N, Kochupillai N. Vitamin D status in primary hyperparathyroidism in India. Clin. Endocrinol (Oxf). 1995:43:351-58.

[4] Goswami R, Gupta N, Goswami D, Marwaha RK, Tandon N, Kochupillai N. Prevalence and significance of 25-hydroxyvitamin $D$ concentrations in healthy subjects in Delhi. Am J Clin Nutr. 2000;72:472-75.

[5] Harinarayan CV, Sachan A, Reddy AP, Satish KM, Prasad UV, Srivani P. Vitamin D status and bone mineral density in women of reproductive and postmenopausa age groups: A cross-sectional study from south India. J Assoc Physicians India. 2011;59:695-701

[6] Marwaha RK, Tandon N, Garg MK, Kanwar R, Narang A, Sastry A, et al. Vitamin D status in healthy Indians aged 50 years and above. J Assoc Physicians India. 2011;59:703-07

[7] Gröber U, Spitz J, Reichrath J, Kisters K, Holick MF. Vitamin D: Update 2013 from rickets prophylaxis to general preventive healthcare. Dermato-Endocrinology. 2013;5(3):e2,331-47

[8] Pittas AG, Sun Q, Manson JE, Dawson-Hughes B, Hu FB. Plasma 25hydroxyvitamin $\mathrm{D}$ concentration and risk of incident type 2 diabetes in women. Diabetes Care. 2010;33:2021-23

[9] Thorand B, Zierer A, Huth C, Linseisen J, Roden M, Peters A, et al. Effect of serum 25-hydroxyvitamin $D$ on risk for type 2 diabetes may be partially mediated by subclinical inflammation: Results from the MONICA/RORA Ausburg study. Diabetes Care. 2011;34:2320-22.

[10] Rock CL, Emond JA, Flatt SW, Heath DD, Karanja N, Pakiz B, et al. Weight loss is associated with increased serum 25-hydroxyvitamin $\mathrm{D}$ in overweight or obese women. Obesity (Silver Spring). 2012;20:2296-301.

[11] Wacker M and Holiack, MF. Vitamin D-effects on skeletal and extraskeletal health and the need for supplementation. Nutrients. 2013;5(1):111-48.

[12] Goswami R, Gupta N, Ray D, Singh N, Tomar N. Pattern of 25-hydroxy vitamin $\mathrm{D}$ response at short (2 month) and long (1 year) interval after 8 weeks of ora supplementation with cholecalciferol in Asian Indians with chronic hypovitaminosis D. Br J Nutr. 2008;100:526-29. 
[13] Allam RR, Pant R, Uthappa CK, Dinaker M , Oruganti G, Yeldandi W. Prevalence of Vitamin D deficiency, metabolic syndrome and association between the two in a South Asian Population. J Nutr Disorders Ther. 2018;8(2):229-34.

[14] Muley A, lyer U. A pilot study on vitamin D status and metabolic syndrome in adult Indian population. Int J Appl Sci Biotechnol. 2014;2(2):126-31.

[15] Talwalkar PG, Deshmukh V, Deepak MC, Agrawal D, Patel I, Hegde R. Prevalence and clinico-epidemiology of vitamin $D$ deficiency in patients with type 2 diabetes mellitus and hypertension- A Pan-India study. Endocrine Abstracts. 2018;56:218.

[16] WHO Physical status: The use and interpretation of anthropometry. World Health Organisation, 1995 (Editor ed.^eds.).

[17] National Institutes of Health, National Heart, Lung, and Blood Institute. Clinical guidelines on the identification, evaluation, and treatment of overweight and obesity in adults; the evidence report. Obes Res. 1998;6(suppl 2):51-209S.

[18] Springbett P, Buglass S, Yo AR. Photoprotection and vitamin-D status. J. Photochem Phobiol B. 2010;101:160-68.

[19] Lips P. Vitamin D deficiency and secondary hyperparathyroidism in the elderly: Consequences for bone loss and fractures and therapeutic implications. Endocr Rev. 2001;22:477-501.
[20] Grundy SM. Diagnosis and Management of the Metabolic Syndrome: An American Heart Association/National Heart, Lung, and Blood Institute Scientific Statement: Executive Summary. Circulation. 2005;112(17):e285-90.

[21] Taheri E, Saedisomeolia A, Djalali M, Qorbani M, Madani Civi M. The relationship between serum 25-hydroxy vitamin $D$ concentration and obesity in type 2 diabetic patients and healthy subjects. J Diabetes Metab Disor. 2012;11(1):16.

[22] Maged MY, Abed El-Raoof DM, Mohammed MY. Serum vitamin D status in type 2 diabetic patients from Gaza Strip. Diabetes Metab Syndr. 2019;13(3):1865-70.

[23] Subramanian A, Nigam P, Misra A, Pandey RM, Mathur M, Gupta R, et al. Severe vitamin $D$ deficiency in patients with type 2 diabetes mellitus in north India. Diabetes Manage. 2011;1(5):477-83.

[24] Holick MF, Chen TC. Vitamin D deficiency: A worldwide problem with health consequences. Am J Clin Nutr. 2008;87(4):1080S-6S.

[25] Shamaila R, Per BJ. Body mass index, vitamin D, and type 2 diabetes: a systematic review and meta-analysis. Nutrients. 2018;10(9):1182.

[26] Bahareh N, Majid H, Amir-Hossein Y, Zahra A, Mahnoosh S, Amaneh MD, et al. Hypovitaminosis $D$ in adults living in a sunny city: relation to some cardiometabolic risk factors, national food and nutrition surveillance. Nutr Food Sci Res 2018:5(1):09-14.

\section{PARTICULARS OF CONTRIBUTORS}

1. Assistant Professor, Symbiosis School of Biological Sciences, Symbiosis International (Deemed) University, Pune, Maharashtra, India

2. Professor, Department of Foods and Nutrition, Faculty of Family and Community Sciences. The M. S. University of Baroda, Vadodara, Gujarat, India.

\section{NAME, ADDRESS, E-MAIL ID OF THE CORRESPONDING AUTHOR:}

Dr. Arti Saurabh Muley,

Symbiosis School of Biological Sciences, Symbiosis International (Deemed University), Symbiosis Knowledge Village Lavale, Pune-412115, Maharashtra, India.

E-mail: arti.muley@ssbs.edu.in

Date of Submission: May 13, 2019 Date of Peer Review: Jun 08, 2019

Date of Acceptance: Jul 07, 2019

FINANCIAL OR OTHER COMPETING INTERESTS: As declared above.
Date of Publishing: Oct 01, 2019 